\title{
El impacto del cambio en el desarrollo de colecciones en bibliotecas universitarias
}

\author{
María del Carmen Negrete Gutiérrez \\ Centro Universitario de Investigaciones Bibliotecológicas \\ UNAM
}

\subsection{Resumen}

El desarrollo de la colección dentro de las bibliotecas universitarias es una tarea que cobra creciente importancia entre los profesionales de la información, puesto que se ha convertido en el marco dentro del que se interrelacionan las diferentes funciones y actividades que lleva a cabo la biblioteca. Se definen y analizan los factores internos y externos que inciden sobre esta tarea. Finalmente, se propone un cuestionario que permite diseñar una estrategia según la cual la institución desarrolla su colección aunando a la adquisición de fondos la participación en programas de adquisición cooperativa y el acceso a servicios complementarios en línea.

Palabras clave: Desarrollo de colecciones. Bibliotecas universitarias. Programas de adquisición cooperativa. México.

\subsection{Abstract}

Collection development in the university libraries is a task of growing importance among the information professionals, because it has grown into the frame where the different functions and activities of the library get interrelated. The internal and external factors that affect this task are defined and analyzed. Finally, a questionnaire is presented that allows the implementation of a strategy that considers collection development throughout holdings acquisition, cooperative acquisition and access to online services.

Keywords: Collection development. University libraries. Cooperative acquisition programs. Mexico.

Scire. $5: 1$ (en.-jun. 1999) 55-64. 


\section{Introducción}

Las instituciones de educación superior constituyen parte importante del desarrollo del país dado que están estrechamente vinculadas con el progreso económico, el desarrollo científico y tecnológico y, con las mejoras sociales, educativas y culturales.

Por lo anterior, el papel que juega la biblioteca universitaria es fundamental, ya que representa una fuente de información para apoyar al proceso educativo, el estudio y el desarrollo de la investigación en todas las áreas del conocimiento.

Así, al cumplir con su misión, la biblioteca ha sido por tradición la principal conservadora del conocimiento y de las ideas acumuladas por el hombre en su lucha por conquistar el mundo físico, por relacionarse eficazmente con sus semejantes y por desarrollar sus facultades intelectuales y espirituales.

Actualmente las bibliotecas universitarias y de investigación están viviendo tiempos de profundos cambios, lo cual se observa tanto en el requerimiento de nuevos patrones para el servicio bibliotecario como en la organización y administración del conocimiento. Esto, se deriva del incremento en la producción de información, de su representación en diversos soportes impresos y electrónicos, y de la variedad de costos que tiene cada uno de ellos. Aunado a todo ello se da también una diversidad creciente de usuarios con necesidades y demandas de información cada vez mayores y más especializadas.

Todas estas presiones internas y externas de la biblioteca, juegan un papel sumamente importante en el desarrollo de colecciones y en el proceso de selección de materiales, y obligan a los bibliotecólogos y profesionales de la información a revisar sus estrategias tradicionales para formar y orientar sus colecciones.

En el caso concreto de la biblioteca universitaria mexicana, esta no puede ser ajena a la época que estamos viviendo, la cual, por un lado se ha vuelto de alta competitividad dada su entrada a un mercado internacional donde la información juega un papel determinante que influye en el progreso, desarrollo y modernización, mientras que por el otro está inmersa en la coyuntura económica que vive el país, lo que causa una inmediata repercusión en las bibliotecas debido, sobre todo a la continua baja en el poder adquisitivo de nuestra moneda, lo cual dificulta la adquisición de materiales.

El reto que representa lo anterior exige una conciencia clara y una decisión de trabajo, así como un compromiso por parte de los sectores responsables del desarrollo de colecciones en este tipo de bibliotecas.

La inquietud por desarrollar, mantener y acrecentar la calidad, cantidad y diversidad de información en los acervos, ha sido motivo de preocupación constante en las bibliotecas universitarias, las cuales ahora se enfrentan a los avances 
científicos y tecnológicos que vienen aportando un vasto campo de acción al proceso de desarrollo de colecciones, lo que sin lugar a dudas está produciendo una concepción más amplia del proceso y de las posibilidades que conlleva la formación y orientación de colecciones.

Ante estos fenómenos es urgente que el bibliotecólogo y/o los profesionales de la información ubicados en las bibliotecas universitarias desarrollen un entendimiento claro y evolutivo de su papel, pues sólo así podrán optimizar sus recursos informativos, económicos y humanos, y enfrentar las complejidades que impone la información misma y sus nuevos medios de acceso, los cuales están cambiando el cómo aprender, el cómo comunicarse, el cómo investigar y el cómo desarrollar las colecciones.

\section{El desarrollo de colecciones}

Edward Evans define el desarrollo de colecciones como el proceso de satisfacer las necesidades de información de la comunidad de manera oportuna y económica, usando tanto los recursos de información que se poseen localmente como los de otras organizaciones. (Evans, 1995, p. 17).

En la definición señalada, el autor deja de lado aspectos de suma importancia con respecto a las colecciones, por lo que, sin dejar de reconocer su idea principal, el desarrollo de colecciones se define como el proceso que permite satisfacer las necesidades y demandas de información de la comunidad mediante la formación y orientación de colecciones básicas y fortalecidas, tanto en alcance como en profundidad en todas las áreas y temas de interés de manera sistemática y coherente, y que puede complementarse con el acceso y/o disponibilidad de aquellos recursos que se localizan fuera de la biblioteca.

Es indudable que esas necesidades deben ser vistas principalmente en el contexto de los programas educativos que se imparten y en los proyectos de investigación que se desarrollan para la institución a la que la biblioteca debe apoyar. De ahí que el objetivo del desarrollo de colecciones en la biblioteca universitaria sea formar y orientar una colección de materiales que se ajuste al objetivo de la institución de la que depende, así como al perfil de necesidades y demandas de información de su comunidad, manteniendo un adecuado balance cualitativo y cuantitativo entre las diferentes áreas de interés y entre los diversos tipos de materiales representados en los distintos formatos (Negrete, 1988, p.10).

El desarrollo de colecciones es un área fundamental de la misión de la biblioteca, la cual recientemente ha cobrado mayor interés entre los bibliotecólogos y los profesionales de la información, principalmente entre quiénes reconocen que formar y orientar una colección se ha vuelto hoy una actividad sumamente compleja debido a que diversos factores externos a la biblioteca no sólo han influido

Scire. $5: 1$ (en.-jun. 1999) 55-64. 
en el incremento de las demandas de información de los usuarios, sino que también están modificando la conceptualización tradicional del desarrollo de colecciones, su objetivo y los procesos que requiere su adecuado manejo.

Con lo anterior, el proceso de desarrollar colecciones debe ser considerado como el marco donde se enclavan e interrelacionan todas las funciones y actividades que se realizan en la biblioteca (factores internos), y que conforman su estructura organizativa para cumplir con sus metas y objetivos. Todo esto sin dejar de lado el estudio de las influencias externas a la biblioteca, es decir : el comportamiento editorial e informativo; los recursos ubicados en otras bibliotecas o instituciones; y el surgimiento de diversos medios electrónicos que permiten una mayor diseminación y transferencia de la información, hecho al que no es ajena la comunidad universitaria, quien está más familiarizada que en años anteriores con las posibilidades que ofrecen los nuevos medios electrónicos, con las diversas formas en que se presenta la información, y con los diferentes servicios que permiten la diseminación y accesibilidad al conocimiento.

Ante este panorama, es necesario tener una visión integral del proceso que considere los factores internos y externos a la biblioteca, los cuales se complementan e interrelacionan de forma cíclica y permanente.

Los factores internos mas comunes se pueden agrupar en ocho grandes rubros:

- El objetivo de la biblioteca

- El estudio de la comunidad

- La evaluación de la colección

- Los recursos disponibles

- Los servicios bibliotecarios

- El presupuesto

- La selección de materiales

- La adquisición de materiales

Dentro de los factores externos están :

- La cooperación bibliotecaria

- El mercado editorial e informativo

- La dinámica de la información

- Los desarrollos tecnológicos

- Los productores y distribuidores (Negrete, 1998b)

El proceso de desarrollo de colecciones va más allá de las actividades de selección y adquisición de materiales, dado que implica un momento de planea- 
ción al cual se le debe, allegar, por principio, información clara y actual sobre todos y cada uno de los elementos o factores apuntados, así como el análisis de cada uno de ellos. Esto debe involucrar una investigación sobre los procesos en sí mismos y no solamente sobre los procedimientos efectuados en cada uno de ellos, lo que permitirá obtener un diagnóstico de la situación actual en que se encuentra la biblioteca a la vez proporcionará una apropiada organización para desarrollar colecciones con una visión a futuro y dentro del marco de nuestras realidades económicas, políticas, sociales, y culturales.

En el marco de la realidad mexicana (que podría ser también la de algunos países latinoamericanos), planear el desarrollo de colecciones e implementar nuevos medios de servicio en la biblioteca universitaria debe implicar un proceso que esté relacionado con la realidad en que se inscribe y que tome en consideración todos los elementos que influyen y determinan la toma de decisiones en el proceso de selección.

Probablemente en países desarrollados algunos factores no determinen este proceso de la misma manera en que nuestros países y por tanto no tendrían porque considerarlos. Tal sería el caso del presupuesto, los desarrollos tecnológicos, los recursos disponibles, la cultura, etc.

En la última década, se observa con frecuencia en la literatura especializada, afirmaciones como ésta:

El desarrollo y la organización de las colecciones están cediendo inevitablemente ante el acceso a la información y a los recursos de la información, independientemente del lugar donde se encuentren. Los recursos de información en forma física, cederán su lugar a los datos computarizados y a las imágenes electrónicas. (Malinconico, 1993)

Lo señalado por el autor no podría tomarse como una generalización válida para toda biblioteca universitaria, dado que cada una de ellas responde a realidades diferentes y con características específicas. Sin embargo las bibliotecas no pueden ser ajenas a los procesos de cambio que se dan en su entorno y deben reconocer los beneficios que conlleva el acceso a la información y a la satisfacción de estas necesidades y demandas de su comunidad.

Ahora vemos que a un mayor número de usuarios universitarios, no les interesa saber por qué los recursos no se localizan en la biblioteca; lo que quieren y demandan son respuestas a sus necesidades de información. Dichas actitudes se deben, en parte, a la influencia ejercida por la naturaleza de los servicios que ahora se ofrecen en algunas bibliotecas universitarias; aunado a una mayor difusión a través de los diversos medios de comunicación, sobre las bondades que conllevan las modernas tecnologías para el acceso a la información.

Scire. $5: 1$ (en.-jun. 1999) 55-64. 
Es una realidad que el acceso a la información, independientemente de donde ésta se localice, viene a resolver carencias de indudable importancia en nuestras bibliotecas. La presencia de medios electrónicos como Internet y el correo electrónico han tenido un impacto a nivel mundial y han permitido establecer canales y flujos de información cada vez más poderosos entre los individuos, entre diversas instituciones y entre los proveedores de información. Las listas de interés, por ejemplo, han abierto un camino para la comunicación académica, la cooperación interbibliotecaria y la cooperación entre las personas interesadas en satisfacer necesidades concretas de sus usuarios o en resolver problemas planteados por colegas.

Ahora tenemos mayor acceso a más información y con mayor rapidez. Esto no implica necesariamente que toda la información a la que tenemos acceso por medios electrónicos sea de calidad, ni tampoco que toda la comunidad en cualquier institución tenga acceso a la información electrónica, ya que "el acceso a la información electrónica, es para quien tiene acceso a una computadora que esté conectada a redes nacionales y mundiales, que pueda leer en inglés principalmente, por tanto no es disponible para todos, no es tan democrático el acceso, ni mucho menos gratuito" (1).

Además de lo anterior habría que considerar que la transferencia de información implica contar con amplios o suficientes presupuestos, equipo actualizado, diversos programas, tiempo de acceso a diversos servicios y productos que generan u ofrecen los productores y, principalmente, obliga a contar con personal profesional que conozca y apoye los procesos de acceso para la localización, búsqueda, selección y obtención de la información relevante y adecuada para las necesidades de información.

Ante estas ideas generales se debe tener presente que la biblioteca debe planear el desarrollo de colecciones con el objeto principal de satisfacer las necesidades de información de su comunidad en el marco de la realidad en que se inscribe, y sin perder de vista que “... las tendencias apuntan a que será práctica común en mantener una colección mixta, es decir un núcleo en papel complementado por el acceso a documentos electrónicos" (Pérez y Cadavid, 1996).

Aunque nadie puede discutir la importancia que tiene el hecho de que la información que se necesita en un momento dado esté disponible en la estantería de la propia biblioteca, es necesario considerar que frente al crecimiento de las necesidades de información y, al mismo tiempo frente al crecimiento de la información producida, es cada vez más difícil que una sola biblioteca posea todos los materiales de información que su comunidad demanda. Por tanto es necesario considerar fuentes alternativas para obtener la información. 
Actualmente en la literatura de la especialidad, diversos autores señalan la confrontación que existe entre "propiedad vs. acceso" a la información, entendiendo por la primera la posibilidad de que la información esté en los propios estantes de la biblioteca, mientras que por la segunda se supone que nos referiríamos a una información disponible normalmente a través de un medio o a través de la obtención misma del documento.

Hoy es relativamente fácil saber que materiales tiene no solamente la biblioteca más cercana, sino también la más lejana. El problema es ahora cómo encontrar el adecuado equilibrio entre poseer colecciones dentro de la propia biblioteca y tener acceso a la información que existe fuera de ésta. Ambos términos no son necesariamente incompatibles, aunque un adecuado balance entre ellos supone una medición sobre lo exitosa que ha sido la colección de una biblioteca para atender las necesidades concretas de su comunidad, y cuáles de esas necesidades, al no ser posiblemente tan urgentes, pueden ser atendidas con base en las colecciones de otras bibliotecas o cuáles de esas necesidades al ser posiblemente urgentes pueden ser atendidas con el acceso electrónico. Es lógico que quien coordina el desarrollo de colecciones en la biblioteca universitaria tenga que prestarle una gran atención a la demanda específica que se ejerce sobre cierto tipo de materiales, como sería el núcleo intelectual de cada disciplina o materia y el núcleo básico de obras de consulta. Colecciones del tipo mencionado, deben ser parte del acervo básico de la biblioteca universitaria.

En este contexto, tanto los responsables de coordinar el desarrollo de colecciones como los encargados de la selección de materiales, requieren de amplios conocimientos y experiencia para decidir qué recursos impresos se deben adquirir, cuáles se deben compartir y cuáles de ellos serán consultados a través del acceso electrónico. Esto implica necesariamente el establecimiento de políticas y criterios para seleccionar materiales impresos y para elegir entre varios y diferentes niveles de acceso electrónico a la información.

La biblioteca universitaria debe considerar el acceso y o disponibilidad a la información con base en tres vertientes :

- como una manera de satisfacer necesidades y demandas prioritarias de información.

- como una forma de completar colecciones y ...

- como una manera de hacer sobrevivir a la biblioteca.

En consecuencia la cooperación entre bibliotecas e instituciones juega en esto un papel fundamental. Si bien es cierto que no podemos basar el desarrollo de nuestras colecciones sobre lo que el vecino está comprando, también lo es que mediante el establecimiento adecuado de convenios de cooperación, como sería el caso del préstamo interbibliotecario a nivel institucional o la creación de con-

Scire. $5: 1$ (en.-jun. 1999) 55-64. 
sorcios, las bibliotecas pueden beneficiarse con programas de adquisición compartida o coordinada.

Lo anterior requiere que el personal profesional de las bibliotecas considere como un componente importante del desarrollo de sus colecciones no sólo la cercanía de bibliotecas similares para establecer convenios o programas cooperativos, sino también que haga suya la obligación moral y profesional para desarrollar aquellas colecciones básicas para sus propios usuarios, dado que no sería éticamente válido desarrollar los recursos de una biblioteca considerando en primera instancia los recursos de las bibliotecas vecinas, como ocurre en algunos casos.

Otra manera de acceder a la información se da en las bases de datos en línea que también permiten el posible suministro de documentos. Sin embargo habría que tener presente que el acceso a estos formatos implica un costo que de alguna manera se debe recuperar. Es entonces cuando aparece el dilema de si cobrarle o no al usuario por nuestros servicios especializados. Este debate erosiona sin duda en la misión misma de la biblioteca pública universitaria.

Es ya una realidad en que algunas de estas bibliotecas se recurre cada vez más al acceso remoto cuando sus recursos informativos son insuficientes, lo cual no quiere decir que esto sea aceptable como principio bajo el cual se deben desarrollar las colecciones.

Es un hecho que no se puede hablar de bibliotecas basadas solamente en la propiedad de la información o basadas en el acceso electrónico, pues en ambos casos enfrentarían dificultades insalvables para satisfacer las necesidades y demandas de información de su comunidad universitaria.

La alternativa que se plantea es la biblioteca híbrida, concebida como aquella que tiene los recursos propios que son necesarios para atender a sus usuarios, y que además se asegura de tener los accesos a aquellos servicios que le resultan complementarios.

Actualmente no podríamos hablar de servicios de calidad si no contáramos con la tecnología, lo que no quiere decir que se puedan juzgar tales servicios sin tener un conocimiento real sobre sus usuarios y sus necesidades, así como sobre las fortalezas y debilidades de su colección y sobre las formas y el uso que se hace de ésta.

Adquirir la información o posibilitar su acceso, es una decisión que tiene que responder cuidadosamente a una planeación estructurada sobre la base de cuando menos diez cuestionamientos que a continuación se enuncian (Negrete, 1998a):

1. ¿Qué funciones principales de la institución debe apoyar la biblioteca ? 
2. ¿Qué nivel de necesidades y características reúne su comunidad?

3. ¿Qué tipo y qué nivel de información debe proporcionar la biblioteca?

4. ¿Cuál es el estado actual que guarda la colección en las áreas o temas de interés para la biblioteca?

5. ¿Qué servicios de información requiere adecuar o implementar la biblioteca?

6. ¿Se tiene el apoyo académico y económico institucional?

7. ¿Cómo se debe distribuir el presupuesto para apoyar estos servicios?

8. ¿Se cuenta con personal profesional capaz de proporcionar los servicios?

9. ¿Se obtendrán los documentos físicamente, y cuándo?

10. ¿Quién absorberá el costo de la obtención de los documentos?

Por todo lo anterior estamos de acuerdo en que: "Conjuntar el estudio de las necesidades de información y comportamiento informativo de las comunidades académicas con el análisis de los recursos tecnológicos disponibles para la operación de servicios, puede ayudar a construir una estrategia de corto y largo plazo que evite el sesgar el diseño de servicios únicamente a partir de la oferta de recursos tecnológicos". (Lafuente, 1997)

\section{A manera de conclusión}

El concepto tradicional de desarrollo de colecciones todavía seguirá dominando en la mayoría de las bibliotecas universitarias mexicanas, debido principalmente al papel que juega como apoyo en la enseñanza-aprendizaje y en las necesidades de información y recreación, y por la dificultad de cambiar operaciones basadas en la propiedad de la información a operaciones basadas en el simple acceso electrónico. Sin embargo, es necesario revisar los esquemas que subyacen a las actividades y funciones de la biblioteca y analizar si le permiten cumplir realmente con el objetivo planteado por el desarrollo de colecciones, satisfacer las necesidades y demandas de información de su comunidad.

La planeación del desarrollo de colecciones debe obedecer a un análisis detallado de todos los factores que intervienen en él, y procurar la estructuración de objetivos y metas claramente definidos. Un plan así producido, podrá ser el mejor instrumento para confrontar el cambio tecnológico y para clarificar la confrontación que probablemente no existe entre propiedad y acceso a la información.

\section{Notas}

(1) Mesa redonda sobre "El bibliotecólogo en un ambiente de telecomunicaciones", celebrada en el marco del Primer Seminario Internacional sobre Desarrollo de Colecciones, el día 7 de Nov. de 1997.

Scire. $5: 1$ (en.-jun. 1999) 55-64. 


\section{Bibliografía}

Adams, Judith A. ; Bonk, Sharon C. (1995). Electronic information technologies and resources : use by university faculty and faculty preferences for related library services. // College and Research Libraries. 56 : 2 (March ,1995) 119-131.

Evans G. Edward (1995). Developing Library and Information Center Collections. $3^{\mathrm{a}}$. ed. Englewood, Col. : Libraries Unlimited, 1995.

Evans, G. Edward. (1995). Developing Library and Information Center Collectios. $3^{\mathrm{a}}$. Englewood, Col. : Libraries Unlimited, 1995. 556 p.

Ifidon, Sam E. (1997). Planing for collection development in the twenty-first century. // World Libraries. 7 : 2 (Spring, 1997) 27-39

Lafuente López, Ramiro (1997). En el umbral del cambio : las tecnologías de la información y la comunicación. México : UNAM/CUIB, 1997.

Malinconico, S. Michael (1993). Los servicios de información en la biblioteca electrónica. // Tecnología de la información. 3 : 2 (1993).

Moffat, Catherine (1996). The future of collection development, selection and aquisitions. // The Australian Library Journal. 45 : 3 (August, 1996) 209-214.

Negrete Gutiérrez, María del Carmen (1988). La selección de materiales documentales en el desarrollo de colecciones. México : UNAM/CUIB, 1988.

Negrete Gutiérrez, María del Carmen. (1998a). Consideraciones sobre el desarrollo de colecciones : propiedad vs. acceso a la información en la biblioteca universitaria. // La información en el inicio de la era electrónica. México : UNAM/CUIB, 1998. p. 36

Negrete Gutiérrez, María del Carmen (coord.) (1998b). Primer Seminario Internacional sobre Desarrollo de colecciones. México : UNAM/CUIB, 1998. 240 p.

Pérez Gómez, Martha Alicia ; Cadavid Arango, Carlos A. (1996). Desarrollo de colecciones propias vs. acceso remoto. // Primer seminario Internacional sobre el Desarrollo de Colecciones. México : UNAM/CUIB, 1997. 